\title{
Expeditious prediction of fiber content in sugar cane: An analytical possibility with LIBS and chemometrics
}

\author{
João Paulo Rodrigues Romera, Paulo Lopes Barsanelli, Fabíola Manhas Verbi Pereira* \\ Departamento de Química Analítica, Instituto de Química, Universidade Estadual Paulista “Júlio de Mesquita Filho" (Unesp), Rua Professor Francisco Degni, 55, Araraquara, SP \\ 14800-060, Brazil
}

\section{A R T I C L E I N F O}

\section{Article history:}

Received 17 August 2015

Received in revised form 4 November 2015

Accepted 8 November 2015

\section{Keywords:}

Bioenergy

Fiber content

Sugar cane

Chemometrics

LIBS

\begin{abstract}
A B S T R A C T
The development of a multivariate model for fiber content prediction in sugar cane has been investigated and can be offered as an alternative to the wet methods of analysis. The importance of fiber in bagasse of sugar cane is directly linked to the production of sugar and alcohol performances and to payment systems. The analytical method investigated here was the Laser-Induced Breakdown Spectroscopy (LIBS) combined with a Partial Least Squares (PLS) chemometric tool that can achieve the best model to predict fiber content in sugar cane. A total of 6287 spectra of raw samples of sugar cane bagasse was evaluated. The optimum conditions of operation of the equipment for the acquisition of the most sensitive LIBS signals were tested through Design of Experiments (DOE).
\end{abstract}

(c) 2015 Elsevier Ltd. All rights reserved.

\section{Introduction}

The content of fiber in sugar cane is one of the main parameters to evaluate the yield of the final product and is directly related to the quantity of the juice supply to manufacture two important commodities - sugar and ethanol [1].

The potentialities of Laser-Induced Breakdown Spectroscopy (LIBS) applications are wide for analytical purposes, such as directly performing analysis in solid samples, thus saving time and without being necessary intense sample preparation [2]. The information provided by LIBS spectra is huge and the spectroscopy signals are better exploited with chemometric tools [3-5]. Then, the goal of this research is the development of an alternative method with high sample throughput for directly prediction of fiber content in sugar cane.

In order to contribute to this demand, the fiber content stands out as one of the main parameters of sugar cane that are routinely monitored as consignment for payment purposes, since the extraction of juice depends on the physical separation of the fiber process (bagasse). In other words, the amount of fiber present in sugar cane can be directly related to the amount of supply juice, and with the following yields of the final products, sugar and ethanol [1].

Sugar cane leaves were also investigated by LIBS for the determination of macro ( $\mathrm{P}, \mathrm{K}, \mathrm{Ca}$ and $\mathrm{Mg}$ ) and micronutrients

\footnotetext{
* Corresponding author. Tel.: +55 1633019609.

E-mail address: fabiola@iq.unesp.br (F.M.V. Pereira).
}

( $\mathrm{B}, \mathrm{Cu}, \mathrm{Fe}, \mathrm{Mn}$ and $\mathrm{Zn}$ ), however the samples were prepared using cryogenic mill and pressed as pellets to perform LIBS mapping [6]. This procedure improves the sensitivity of the data, but it is not attractive to in loco measurements, for instance.

The LIBS technique contribution for this goal is to allow direct measurements of conductive or non-conductive samples, regardless of the physical state (solid, liquid or gas) as well as in aerosol form [2]. The principle of the LIBS is based on the use of a laser as the energy source to promote vaporization of the sample, to excite the emission of electromagnetic radiation from its elements and/or molecular fragments [7]. In this case, one or more emission lines represent the analytical signal of the excited species as a spectrum where the axis of abscissa and ordinate are the corresponding wavelength range and the intensity of emission lines, respectively [2].

Among the potential features of LIBS for the study of this investigation, the following can be indicated: allowing mappings in the samples in different positions; achieving wide spectral range (from ultraviolet to near infrared, in accordance with the settings for each system) and providing organic and inorganic simultaneous characterization [8].

Another advantage is the possibility to perform the analysis without pretreatment or a minimum amount of samples enhancing speed and leading to lower cost analyzes $[9,10]$. These features can point LIBS as a technique that meets the principles of green chemistry, reducing analysis time, decreasing the consumption of reagents in sample pre-treatment without waste generation. 
a

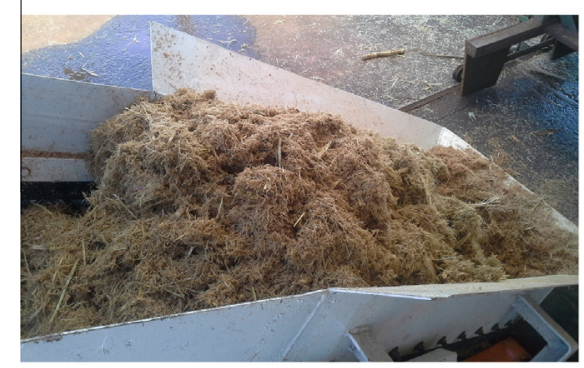

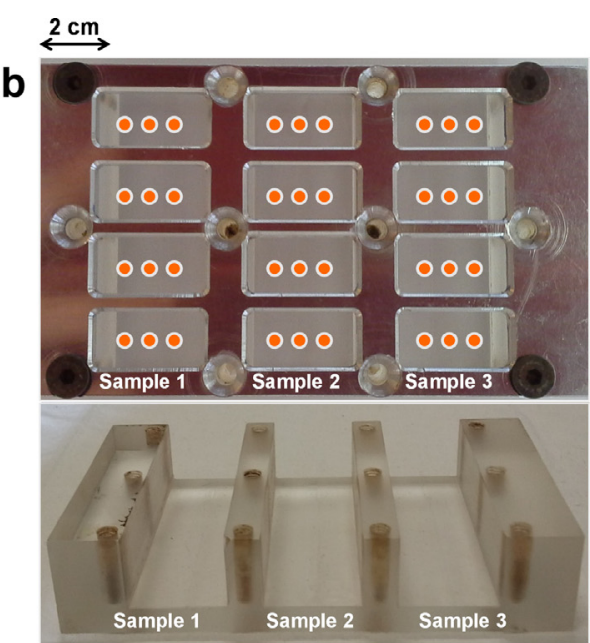

Fig. 1. Picture of raw sugar cane bagasse (a) and pictorial of sample holder used for LIBS measurements of this material.

Table 1

Investigated LIBS parameters using DOE.

\begin{tabular}{llllr}
\hline Test & Laser energy $(\mathrm{mJ})$ & Delay time $(\mu \mathrm{s})$ & Spot size $(\mu \mathrm{m})$ & \multicolumn{1}{c}{ Norm } \\
\hline 1 & $-1(50)$ & $-1(0.5)$ & $-1(75)$ & 6382 \\
2 & 1 & -1 & -1 & 21,073 \\
3 & -1 & 1 & -1 & 2120 \\
4 & 1 & 1 & -1 & 4320 \\
5 & -1 & -1 & 1 & 9515 \\
6 & 1 & -1 & 1 & 23,594 \\
7 & -1 & 1 & 1 & 3159 \\
8 & $+1(75)$ & $+1(1.0)$ & $+1(125)$ & 11,863 \\
\hline
\end{tabular}

Other features make it an attractive LIBS technique also for experiments in the field or at remote distance [11] systems, among which, the configuration of a LIBS system enables the portability of equipment for on-site measurements [12].

Some relevant factors are part of the excitation and emission process and should be considered in studies with LIBS. The first is related to the interaction between the laser and the sample; the second corresponds to that between the laser and material generated by ablation (electrons, molecules, atoms, ions and fine particles) and finally; the remaining pulse energy. The combination of these three factors is essential for the excitation and emission spectral of the chemical elements. It is noteworthy that the phenomena that occur between the laser interaction and the sample can be summarized in the sample vaporization, dissociation, heating and mass transfer and finally, in the ionization and excitation of the material that has been generated $[13,14]$.

The information from spectra generated by LIBS is broad and can be further explored with the application of chemometric tools for the development of multivariate models [15]. Through these models, it would be possible to select atomic lines and/or ionic specific elements that are effectively contributing to the determination of fiber in sugar cane.

In this context, this study aimed to develop an innovative analytical method to predict the fiber content in sugar cane. Data from these multivariate models can derive sugar cane quality parameters, implement automated control of analysis and develop alternative payment systems.

\section{Materials and methods}

\subsection{Samples}

Raw samples of sugar cane bagasse (Fig. 1a) with fiber content ranging up $10-22 \%(\mathrm{~g} / 100 \mathrm{~g})$ were kindly provided by a sugar factory Tonon Bioenergia S/A that is located in the Bocaina city, state of São Paulo. The fiber content was determined according to the recommendations from the Manual of the Council of the producers of sugar cane, sugar and ethanol in the state of São Paulo (CONSECANA) [16] at the quality control laboratory of the sugar factory.

\subsection{LIBS tests}

A dedicated sample holder was manufactured in a mechanic workshop to measure this type of samples, as show in Fig. 1b. The base was made of acrylic with 3 cavities able to place up to three different samples of the sugar cane bagasse. A cover made of aluminum was also machined to compress the sample and to guide the analyst about the mapping positions. The dimensions (in $\mathrm{cm}$ ) of the base were 10.5 (width) $\times 6.0$ (length) $\times 2.0$ (height) The width and the depth (in $\mathrm{cm}$ ) of the cavities were 2.0 and 1.6 , respectively.

\subsection{LIBS data}

The signals were recorded using a commercial J200 LIBS system from Applied Spectra (Fremont, California - USA). The Aurora software package (Applied Spectra) was used for the spectral lines information of each element. A video was included as Supplementary material to better explain the tests with LIBS. After recording, the spectra were normalized with norm unit equals to 1.00 and mean-centered. The preliminary evaluation was performed with Principal Component Analysis (PCA), the model for prediction of fiber content was computed by Partial Least Squares (PLS) using the same transformation and pre-processing described for PCA evaluation. These chemometric tools are available in the Pirouette 4.5 rev. 1 (Infometrix, Bothel, USA) software package.

\section{Results and discussion}

The first task of this study was properly carried on the samples for measurements. Since our method intend to minimal preparation of the samples and at the same time, the material is very heterogeneous (Fig. 1a). Subsequently, a sample holder (Fig. 1b) was set up to improve analytical frequency and compact the samples inside in order to prevent their spreading after the incidence of pulse laser. This sample holder is introduced inside the ablation chamber for recording the emission signals. 


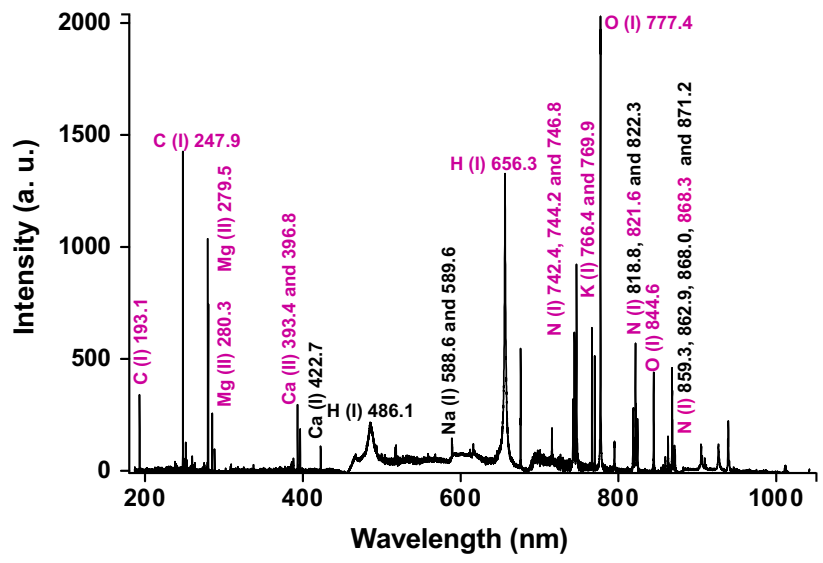

Fig. 2. Profile of average LIBS spectrum for sample with $20 \%$ fiber content. Symbols (I) and (II) represent atomic and ionic lines, respectively. The important lines were purple highlighted.

The sample treatment was avoided, since the frequency of the arrival of the cane bagasse in the sugar factory is very high. The task of this study was to measure the samples as close as possible from the reality of the sugar factory. In the same direction, the moisture control and the pelletizing of the samples were not considered. According to our tests, the influence of moisture did no interfere in the intensity of emission lines and the heterogeneity of samples was compensated with the average of mapped points and the number of laser pulses in each point.

For LIBS measurements, at least three points were randomly mapped per rectangle (see orange circles at Fig. 1b) considering that randomization was necessary to effectively assess this material. After the mapping, an average spectrum was calculated for each sample following the procedure preconized by Galbács [17].

A total of 53 samples with a fiber content in the range between $10 \%$ and $22 \%$ was measured with the LIBS technique, generating a data array with 5327 rows corresponding to the samples and 12,288 columns associated with instrumental response variables $(186.9-1042.0 \mathrm{~nm})$.

With the Design of Experiments (DOE) it was possible to evaluate the influence of three variables in instrumental measures to the LIBS, from low to high levels: (i) 50 and 75 laser energy (mJ), 0.5 and 1.0 delay time ( $\mu \mathrm{s})$ and, 75 and 125 spot size $(\mu \mathrm{m})$. The type of DOE was a full factorial $2^{3}$, i.e. two levels with 3 variables under test, resulting 8 experiments.

The ranges described above cover the possibilities in our commercial LIBS system and further optimization was not possible to be performed. In the case of variable laser energy, it was not tried to work with energies higher than $75 \mathrm{~mJ}$, since the fiber is ruptured due to the high fluence of the laser.

In response to the DOE, the norm value for each of the eight experiments was calculated. The best experimental condition would be the one with the highest value of the norm, the conclusion was that this represented the signals that were generated with the largest number of emission lines and concomitantly with the highest intensities. Eq. (1) provides the norm value.

$\|b\|=\sqrt{b_{1}^{2}+b_{2}^{2}+\cdots+b_{n}^{2}}$

where $b_{1}, b_{2}, b_{3}, b_{n}$ are instrumental responses obtained at each wavelength from LIBS signals.

The experimental design matrix can be seen in Table 1 , where the values are encoded in -1 to +1 from low to high levels. Thus,
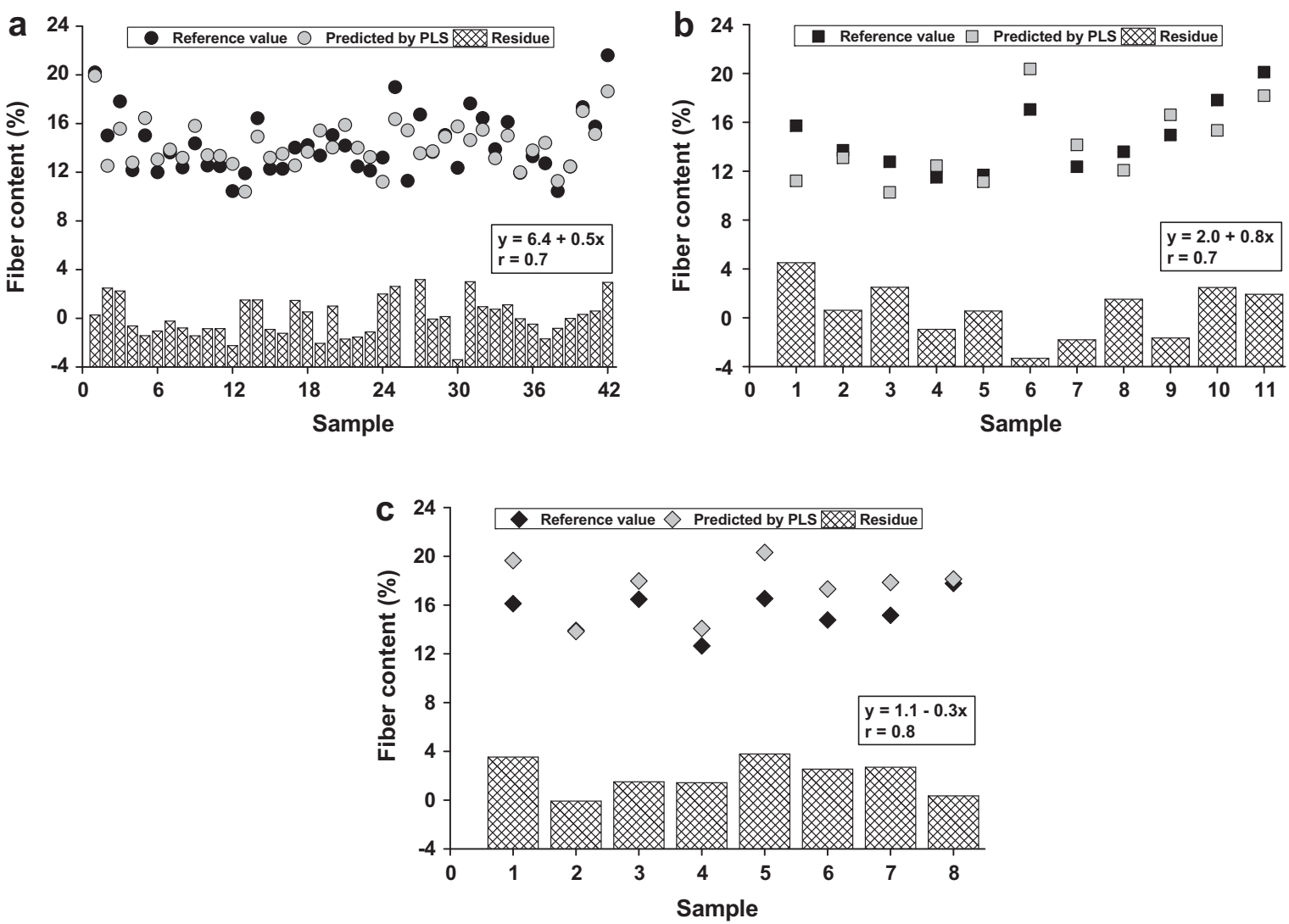

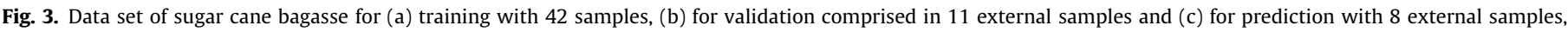
15 months later of the earlier model. 
the LIBS measurements were carried on with the optimized conditions by means of DOE. The best conditions for instrument operation were indicated by the tests 2 and 6 , where the spot size did not influence significantly the response (low effect). Then, the conditions of test 2 were adopted for the measurements, considering higher fluence of the laser promoted by a lower spot size.

Fig. 2 shows a typical spectrum for raw material of sugar cane bagasse. The lines were very reproducible for the samples, independent of the fiber content values. The symbols (I) designate the atomic lines for $\mathrm{C}, \mathrm{H}, \mathrm{O}, \mathrm{N}, \mathrm{Na}$ and $\mathrm{K}$ and (II) ionic lines for $\mathrm{Ca}$ and $\mathrm{Mg}$.

This material has in its composition besides sugar cane, other components such as leaves, straw, soil amendments, set amidst other impurities and moisture. Therefore, the $\mathrm{C}$ atomic line at $193.1 \mathrm{~nm}$ - denoted as C (I), clearly characterizes the fiber content of samples in the presence of these materials.

In order to predict the fiber content with multivariate model, some strategies were tested during the data evaluations. Then, to develop a better model to predict fiber content using the LIBS data from raw samples LIBS and PLS, the wavelengths with regression coefficients higher than 1.0 were chosen. The regression coefficients were computed using a preliminary model with PLS and the entire spectrum (12,288 variables). These values were for the normalized LIBS signals, corresponding to spectra with unitary norm equals to 1.0. This criterion was based on the C (I) at $193.1 \mathrm{~nm}$, being a very important line that was very consistent for all samples.

The data matrix for PLS multivariate model was composed by two data sets, one with 42 samples and 119 variables designated as a training data set and another comprised in external samples with 11 samples and 119 variables called validation data set. The Kennard-Stone algorithm [18] was applied to select a subset of samples for the model. The range with 119 variables corresponds to important atomic lines of $\mathrm{C}, \mathrm{Mg}, \mathrm{Na}, \mathrm{H}, \mathrm{N}, \mathrm{K}$ and ionic lines of Ca as shown in Fig. 2.

The correlation between the reference values from the standard method [16] and those PLS predicted values is very good, since the material was not pretreated and with moisture content, in addition the measurements were performed directly in raw samples. The Root Mean Square Error of Calibration (RMSEC) in \% was 1.8 using the 42 samples of training data set and the RMSE of Validation (RMSEV) was 2.3 for 11 external samples. Five latent variables were chosen which explained $69 \%$ of the variance data. The same magnitude of the errors corroborates as a good indicative to the models potentiality. In the residue values were not verified any heteroscedasticity tendency. The plots of training and validation sets are shown in Fig. 3a and b, respectively.

Fifteen months later, another set of external samples (total of 8) was measured by LIBS under the same previous experimental conditions. The fiber content was predicted with the 53 samples (the data from training and validation sets were combined) using $5 \mathrm{LV}$ representing $67 \%$ of explained variance. The correlation was 0.8 being the residues in the same range of the earlier model and RMSE of Cross Validation (RMSECV) equals to $2.2 \%$, as shown in Fig. 3c.

\section{Conclusions}

The noteworthy of this study was the development of an analytical method of direct analysis to predict fiber content in raw sugar cane bagasse, in the presence of impurities and moisture, without pre-treatment of samples and as close as possible to the nature of the samples. The main contribution is to improve the performance of the production of sugar and alcohol as a consignment for payment purposes.

\section{Acknowledgments}

The authors are grateful to Fundunesp process number 0268/001/14, Grants 2013/04688-7, 2012/50827-6 and 2012/ 01769-3 from São Paulo Research Foundation (FAPESP) and also, Grants 304772/2012-7, 474357/2012-0, 401074/2014-5 and 445729/2014-7 from The National Council for Scientific and Technological Development (CNPq).

\section{Appendix A. Supplementary material}

Supplementary data associated with this article can be found, in the online version, at http://dx.doi.org/10.1016/j.fuel.2015.11.029.

\section{References}

[1] O'Shea MG, Staunton SP, Slupecki P. Int Sugar J 2011:113:879.

[2] Pasquini C, Cortez J, Silva LMC, Gonzaga FB. J Braz Chem Soc 2007;18:463.

[3] Clegg SM, Sklute E, Dyar MD, Barefield JE, Wiens RC. Spectrochim Acta Part B 2009;64:79.

[4] Braga JWB, Trevizan LC, Nunes LC, Rufini IA, Santos Jr D, Krug FJ. Spectrochim Acta Part B 2010;65:66.

[5] Zupan J. J Chemometrics 2015;29:1.

[6] Nunes LC, Braga JWB, Trevizan LC, Souza PF, Carvalho GGA, Santos Jr D, et al. J Anal At Spectrom 2010;25:1453.

[7] Colonna G, Casavola A, Capitelli M. Spectrochim Acta Part B 2001;56:567.

[8] Aragón C, Bengoechea J, Aguilera JA. Spectrochim Acta Part B 2001;56:619.

[9] Pouzar M, Černohorský T, Průšová M, Prokopčáková P, Krejčová A. J Anal At Spectrom 2009;24:953.

[10] Galiová M, Kaiser J, Novotný K, Novotný J, Vaculovič T, Liška M, et al. Appl Phys A 2008:93:917.

[11] Whitehouse AI, Young J, Botheroyd IM, Lawson S, Evans CP, Wright J Spectrochim Acta Part B 2001;56:821.

[12] Wainner RT, Harmon RS, Miziolek AW, McNesby KL, French PD. Spectrochim Acta Part B 2001;56:777.

[13] Sirven JB, Mauchien P, Sallé B. Spectrochim Acta Part B 2008;63:1077.

[14] Galiová M, Kaiser J, Novotný K, Samek O, Reale L, Malina R, et al. Spectrochim Acta Part B 2007:62:1597.

[15] El Haddad J, Canioni L, Bousquet B. Spectrochim Acta Part B 2014;101:171.

[16] CONSECANA - Conselho dos Produtores de Cana-de-Açúcar, Açúcar e Álcool do Estado de São Paulo (Manual of the Council of the producers of sugar cane, sugar and ethanol in the state of São Paulo). Manual de Instruções, 5a Ed., CONSECANA-SP, Piracicaba, São Paulo state; 2006.

[17] Galbács G. Anal Bioanal Chem 2015;407:7537.

[18] Daszykowski M, Walczak B, Massart DL. Anal Chim Acta 2002;468:91. 